\title{
Effects of Water Addition to Prevent Deterioration of Soybean Oil by Calcium Silicate Adsorbent
}

\author{
Fumihiko Ogata ${ }^{1}$, Mikako Obayashi ${ }^{1}$, Eri Nagahashi ${ }^{1}$, Takehiro Nakamura ${ }^{1}$ and \\ Naohito Kawasaki ${ }^{1,2 *}$ \\ ${ }^{1}$ Faculty of Pharmacy, Kindai University, 3-4-1 Kowakae, Higashi-Osaka, Osaka 577-8502, JAPAN \\ ${ }^{2}$ Antiaging Center, Kindai University, 3-4-1 Kowakae, Higashi-Osaka, Osaka 577-8502, JAPAN
}

\begin{abstract}
In this study, we prepared calcium silicate at different molar ratios $(\mathrm{Ca}: \mathrm{Si}=1: 3,1: 6$, and 1:9 refer to CAS-30S, CAS-60S, and CAS-90S, respectively) with water addition. The adsorbent characteristics (specific surface area, pore volume, mean pore diameter, and elemental analysis) were measured and the effect of water addition on the adsorbent surface for the prevention of deterioration was evaluated. In addition, the deterioration of soybean oil (SO) subjected to heating and aeration was investigated based on the acid value (AV) and carbonyl value (CV). The specific surface area increased in the order CAS-60S $\left(160.51 \mathrm{~m}^{2} / \mathrm{g}\right)<$ CAS-30S $\left(182.61 \mathrm{~m}^{2} / \mathrm{g}\right)<$ CAS-90S $\left(204.19 \mathrm{~m}^{2} / \mathrm{g}\right)$. Deterioration of SO could be induced by heating and aeration with $\mathrm{AV}$ and $\mathrm{CV}$ of $1.4 \mathrm{mg} / \mathrm{g}$ and $102.9 \mu \mathrm{mol} / \mathrm{g}$, respectively. The adsorbent (CAS-30S and CAS-90S) with water addition (25\% and $50 \%)$ was found to decrease the AV, indicating that a small amount of water addition to adsorbent surface is important for the decreasing of AV. In addition, the correlation between the decrease in $\mathrm{AV}$ and the specific surface area is strongly positive ( $R$ value: 0.968$)$. The adsorption mechanism is thought to involve interactions between the polar compounds (free fatty acids) in the SO (nonaqueous phase) and the water layer (containing calcium ions released from the adsorbent) on the adsorbent surface. In summary, the data obtained in this study provide useful information for preventing the deterioration of SO and prolonging the oil life cycle.
\end{abstract}

Key words: calcium silicate, soybean oil, adsorption, water addition, deterioration

\section{Introduction}

A large amount of edible oil is wasted domestically, and oily wastewater is produced by many industries. Direct discharge of edible waste oil into drains and water bodies leads to adverse impacts on human health and the environment ${ }^{1)}$. Oil deterioration can be induced by heat, air, and moisture, which lead to polymerization, oxidation, and hydrolysis of the polar compounds ${ }^{2,3)}$. These reactions affect the stability of edible oil. Therefore, it is necessary to develop an environmentally friendly and efficient edible oil treatment and management strategy ${ }^{1)}$. In Japan, 2.29 million tons of edible oil is consumed annually, with the food service industry and domestic use consuming 1.91 and 0.38 tons of oil, respectively, in 2013. Almost all of the waste edible oil is recycled in the food industry, which has a long-established recycling system. In contrast, waste edible oil is almost never recycled domestically. For these reasons, it is necessary to develop recycling techniques for waste edible oil with low material cost and ease of execu- tion.

Several methods have been employed to purify used frying oils ${ }^{4,5)}$. Among these methods, adsorption is widely used because it is low cost and easy to use. Therefore, we focused on the adsorption process for the purification of waste edible oil in this study. Previous studies have reported the use of calcium silicate and magnesium silicate to reduce free fatty acids and manipulate color, respectively ${ }^{6}$. In addition, sepiolite (a hydrated magnesium silicate) was used for the physical refining of degummed rapeseed oil, and it showed high decolorization capacity and sorption capacity (the sepiolite structure contains $\mathrm{Si}-\mathrm{OH}$ groups) ${ }^{7}$. We previously reported that calcium silicate, a food additive, decreased the AV and $\mathrm{CV}$ of deteriorated oil ${ }^{8)}$. These food additives have been proven to be safe for human health and are useful for the purification of waste edible oil. In addition, the application of adsorbent was reported by previous works, which were demonstrated as having ability to control degradation products (polar compounds)

\footnotetext{
*Correspondence to: Naohito Kawasaki, Faculty of Pharmacy, Kindai University, 3-4-1 Kowakae, Higashi-Osaka, Osaka 577-8502, JAPAN

E-mail: kawasaki@phar.kindai.ac.jp

Accepted August 28, 2017 (received for review August 3, 2017)

Journal of Oleo Science ISSN 1345-8957 print / ISSN 1347-3352 online

http://www.jstage.jst.go.jp/browse/jos/ http://mc.manusriptcentral.com/jjocs
} 
by deterioration as well as to remove insoluble particles. Various types of filtration products were investigated, including natural and synthetic adsorbents ${ }^{5}$.

In previous work, water in the nonaqueous phase (oil phase) was shown to be an important factor for the adsorption and removal of free fatty acids ${ }^{9-11)}$. Other studies have reported that the water was adhered onto a polar powder (adsorbent) in the nonaqueous phase, which indicated that the adsorbent interacted with polar compounds in the nonaqueous phase. In addition, the water released from foods affects the deterioration at high temperatures. Moisture migration in adsorption happens when there is a water activity differential between the aqueous phase and nonaqueous phase. The changes in heat of wetting between water and adsorbent surface lead to affect the adsorption capability or removal of polar compounds from aqueous and nonaqueous phase $\mathrm{e}^{11)}$.

Therefore, it is important to investigate the effect of water on the purification of waste edible oil by an adsorbent (calcium silicate) for use as a food additive and environmental cleanup.

The object of the present study was to determine the efficiency of water addition on the purification of waste edible oil. Specifically, we were interested in the removal of polar compounds produced during deterioration by adsorbent treatment with a calcium silicate compound. In addition, the removal mechanism of polar compounds from waste edible oil was investigated.

\section{Material and Methods}

\subsection{Materials}

Edible shirashime soybean oil(SO)was purchased from Showa Sangyo Co., Ltd. Japan, and calcium silicate at different molar ratios $(\mathrm{Ca}: \mathrm{Si}=1: 3,1: 6$, and 1:9 referred to as CAS-30S, CAS-60S, and CAS-90S, respectively)was obtained from Tomita Pharmaceutical Co., Ltd., Japan. The properties of the adsorbents, such as specific surface area, pore volume, mean pore diameter, and elemental composition, were evaluated. Scanning electron microscopy (SEM) images were recorded using a JSM-5200 scanning electron microscope(JEOL, Japan). The specific surface area, pore volume, and mean pore diameter were measured by a NOVA4200e specific surface analyzer(Yuasa Ionics, Japan). The elemental composition was measured by an electron probe micro analyzer(EPMA, JXA-8530F, JEOL, Japan). All additional reagents used in this study were purchased from Wako Pure Chemical Industries, Japan, and used without further purification.

\subsection{Deterioration of SO}

Deterioration of SO was effected by heating untreated $\mathrm{SO}(300 \mathrm{~mL})$ at $180 \pm 10^{\circ} \mathrm{C}$ and spinning at $250 \mathrm{rpm}$ for $7 \mathrm{~h}$ per day, with a total treatment time of $28 \mathrm{~h}$. Deterioration of $\mathrm{SO}$ was evaluated by acid value (AV), carbonyl value (CV), concentration of free fatty acid (FFA), and amount of tocopherol.

$\mathrm{AV}$ and CV were measured by methods described in detail in a previous study. Briefly, the samples $(2.0 \mathrm{~g})$ were dissolved in $100 \mathrm{~mL}$ of an ethanol:ether (1:1) mixture, and the solution was titrated against $0.1 \mathrm{~mol} / \mathrm{L}$ potassium hydroxide with phenolphthalein as the indicator. The AV was calculated according to the titer ${ }^{12)}$. Next, the samples $(0.25$ g) were dissolved in $25 \mathrm{~mL}$ of 1-butanol. Subsequently, 1 $\mathrm{mL}$ of the sample solution and $1 \mathrm{~mL}$ of 2,4-dinitrophenyl hydrazine were mixed for $20 \mathrm{~min}$ at $40^{\circ} \mathrm{C}$. Potassium hydroxide $(8 \% ; 8 \mathrm{~mL})$ was then added to the mixtures, and the samples were centrifuged at $3,000 \times g$ for 5 min in a KUBOTA 3100 centrifuge (KUBOTA Co., Ltd.). The supernatants were analyzed using a UV-1200 spectrophotometer $\left(\right.$ Shimadzu ${ }^{13)}$.

The FFA content was measured as follows. Benzene (0.5 $\mathrm{mL})$ and $14 \%(\mathrm{w} / \mathrm{w})$ boron trifluoride-methanol $(1 \mathrm{~mL})$ were mixed with the samples (10 $\mathrm{mg}$ ), and the suspension was heated for $2 \mathrm{~h}$ at $80^{\circ} \mathrm{C}$. Subsequently, $5 \mathrm{~mL}$ of $n$-hexane and $15 \mathrm{~mL}$ of distilled water were added, and the sample in the $n$-hexane layer was analyzed using a gas chromatograph equipped with a flame ionization detector (GC-2014; Shimadzu). The measurement conditions were as follows: capillary column, FAMEWAX ( $30 \mathrm{~m} \times 0.25 \mathrm{~mm}$ ID; Shimadzu GLC Ltd.); carrier gas, He; column temperature, $200^{\circ} \mathrm{C}$; flow rate, $0.6 \mathrm{~mL} / \mathrm{min}$; and injection volume, $1 \mu \mathrm{L}$. In addition, the tocopherol concentration was estimated by diluting the samples in a mobile phase (hexane:ethyl acetate, 7:3), followed by analysis with high-performance liquid chromatography (HPLC; SPD-10AVP, Shimadzu). The measurement conditions were as follows: column, Inertsil $\mathrm{NH}_{2}(250 \mathrm{~mm} \times 4.6 \mathrm{~mm}$, GL-Science); column temperature, $30^{\circ} \mathrm{C}$; flow rate, $1.0 \mathrm{~mL} / \mathrm{min}$; wavelength of detection, 290 $\mathrm{nm}$; and injection volume, $10 \mu \mathrm{L}$.

\subsection{Adsorbent treatment}

Deteriorated SO was obtained by the method described in section 2.2. The adsorbent $(0.15 \mathrm{~g}$ of CAS-30S, CAS-60S, or CAS-90S) with water was added to the deteriorated SO $(15 \mathrm{~g})$. The suspensions were stirred at $250 \mathrm{rpm}$ for $15 \mathrm{~min}$ at $100 \pm 10^{\circ} \mathrm{C}$, and the filtered through a $0.45 \mu \mathrm{m}$ membrane filter. The filtrated oil was then measured for AV, CV, FFA content, and tocopherol concentration. The adsorbent treated with water was prepared as follows. Purified water $(0.25,0.50,0.75,1.0$, and $1.5 \mathrm{~g})$ was sprayed onto the adsorbent surface $(1 \mathrm{~g})$ and the corresponding water contents of the obtained adsorbent were $25 \%, 50 \%, 75 \%, 100 \%$, and $150 \%$, respectively.

Next, deteriorated SO was prepared by the method described in section 2.2 (a total treatment time of $65 \mathrm{~h}$ ). The obtained deteriorated oil was divided to two groups in this 
study. One group for adsorbent treatment(Treated), and the other without adsorbent treatment (Untreated). Adsorbent treatment was conducted as follows. Adsorbent (2.45 g, CAS-90S) treated with 50\% water addition was added to the deteriorated $\mathrm{SO}(300 \mathrm{~mL})$. The $\mathrm{AV}$ and $\mathrm{CV}$ in the filtered oil were then measured. Similar treatments were repeatedly conducted in duplicate in this $\operatorname{study}\left(1^{\text {st }}: 56-84 \mathrm{~h}\right.$, $2^{\text {nd }}: 84-112 \mathrm{~h}$ ).

\subsection{Measurement of calcium ions released from adsor- bent treated with water}

The adsorbent (0.3 g of CAS-30S, CAS-60S, or CAS-90S) was added to purified water $(15,30$, and $50 \mathrm{~mL})$. The suspensions were shaken at $250 \mathrm{rpm}$, for 5,15 , and $30 \mathrm{~min}$ at $25^{\circ} \mathrm{C}$, and then filtered through a $0.45 \mu \mathrm{m}$ membrane filter. The filtrate was analyzed using inductively coupled plasma atomic emission spectrometry (ICP-OES, iCAP 7600 Duo, Thermo Fisher Scientific Inc., Japan) . The amount released from the adsorbent was calculated using Eq. (1).

$$
r=\left(C_{\mathrm{a}}-C_{\mathrm{i}}\right) V
$$

where $r$ is the amount released $(\mu \mathrm{g}), C_{\mathrm{i}}$ is the concentration before treatment $(\mu \mathrm{g} / \mathrm{L}), C_{\mathrm{a}}$ is the concentration after treatment $(\mu \mathrm{g} / \mathrm{L})$, and $V$ is the solvent volume $(\mathrm{L})$.

\subsection{Statistical analysis}

Statistical differences were evaluated by unpaired Student's $t$-tests; $P$ values less than 0.05 were considered significant. The data are presented as the mean \pm standard deviation.

\section{Results and Discussion}

\subsection{Characteristics of adsorbents (CAS-30S, CAS-60S, and CAS-90S)}

CAS (a food additive) has been approved for use in Japan and has low adsorption ability, poor accumulative properties, and low toxicity in humans. SEM images of the adsorbent are shown in Fig. 1. CAS-30S was confirmed to be spherical in shape, but CAS-60S and CAS-90S were not the same shape in this study. In addition, the adsorbent particle size did not vary, which suggested that the properties of the adsorbents depend on their components (different molar ratios). The physical properties such as specific

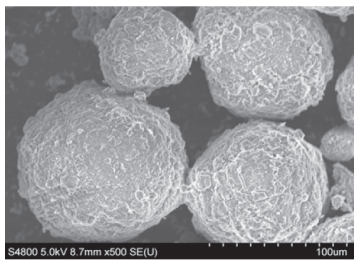

CAS-30S

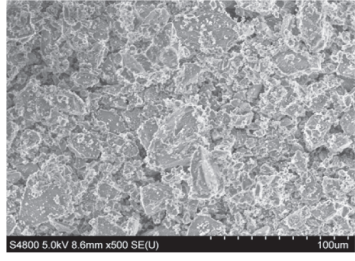

CAS-60S

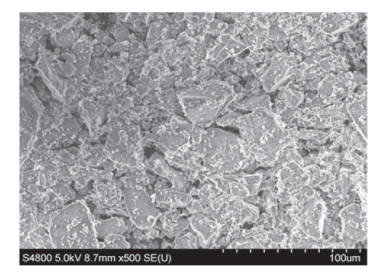

CAS-90S
Fig. 1 SEM images of adsorbents $(\times 500)$.

surface area, pore volume, mean pore dimeter, and elemental composition of the adsorbent surface were also analyzed, and the results are presented in Table 1 and Fig. 2. The specific surface area and pore volume followed the order CAS-60S $<$ CAS-30S $<$ CAS-90S and CAS-90S $<$ CAS-60S $<$ CAS-30S. In addition, the elemental composition of the adsorbent surface was measured (Fig. 2). The calcium content followed the order CAS-90S $<$ CAS-60S $<$ CAS-30S, which indicated that the obtained data agree with the molar contents of the adsorbent, as specified by the manufacturer. CAS was prepared by mixing both $\mathrm{CaO}-$ $\mathrm{SiO}_{2}-\mathrm{H}_{2} \mathrm{O}$ and $\mathrm{CaO}_{1.5}-\mathrm{SiO}_{3.5}-\mathrm{xH}_{2} \mathrm{O}$. The amount of calcium in CAS-30S was greater than that in CAS-60S and CAS$90 \mathrm{~S}^{14)}$.

\subsection{Prepared deteriorated SO}

In this study, we prepared deteriorated SO treated with heating and aeration(Fig. 3). After deterioration, the AV and CV changed from 0.4 to $1.4 \mathrm{mg} / \mathrm{g}$ and 6.5 to 102.9 $\mu \mathrm{mol} / \mathrm{g}$, respectively. The $\mathrm{AV}$ in the deteriorated $\mathrm{SO}$ did not reach a hygienic value $(2.5 \mathrm{mg} / \mathrm{g})$. On the other hand, the $\mathrm{CV}$ in the deteriorated $\mathrm{SO}$ reached a hygienic value (50 $\mu \mathrm{mol} / \mathrm{g}$ ), and the PC content reached a value standardized by the European Union (25.0\%). Therefore, the method used in this study for the deterioration of SO using heat and aeration could be useful for preparing waste edible oil.

Table 1 Properties of adsorbents.

\begin{tabular}{cccc}
\hline Adsorbents & $\begin{array}{c}\text { Specific surface area } \\
\left(\mathrm{m}^{2} / \mathrm{g}\right)\end{array}$ & $\begin{array}{c}\text { Pore volume } \\
(\mathrm{mL} / \mathrm{g})\end{array}$ & $\begin{array}{c}\text { Mean pore diameter } \\
(\AA)\end{array}$ \\
\hline CAS-30S & 182.61 & 0.855 & 176.07 \\
CAS-60S & 160.51 & 0.748 & 180.31 \\
CAS-90S & 204.19 & 0.732 & 149.95
\end{tabular}



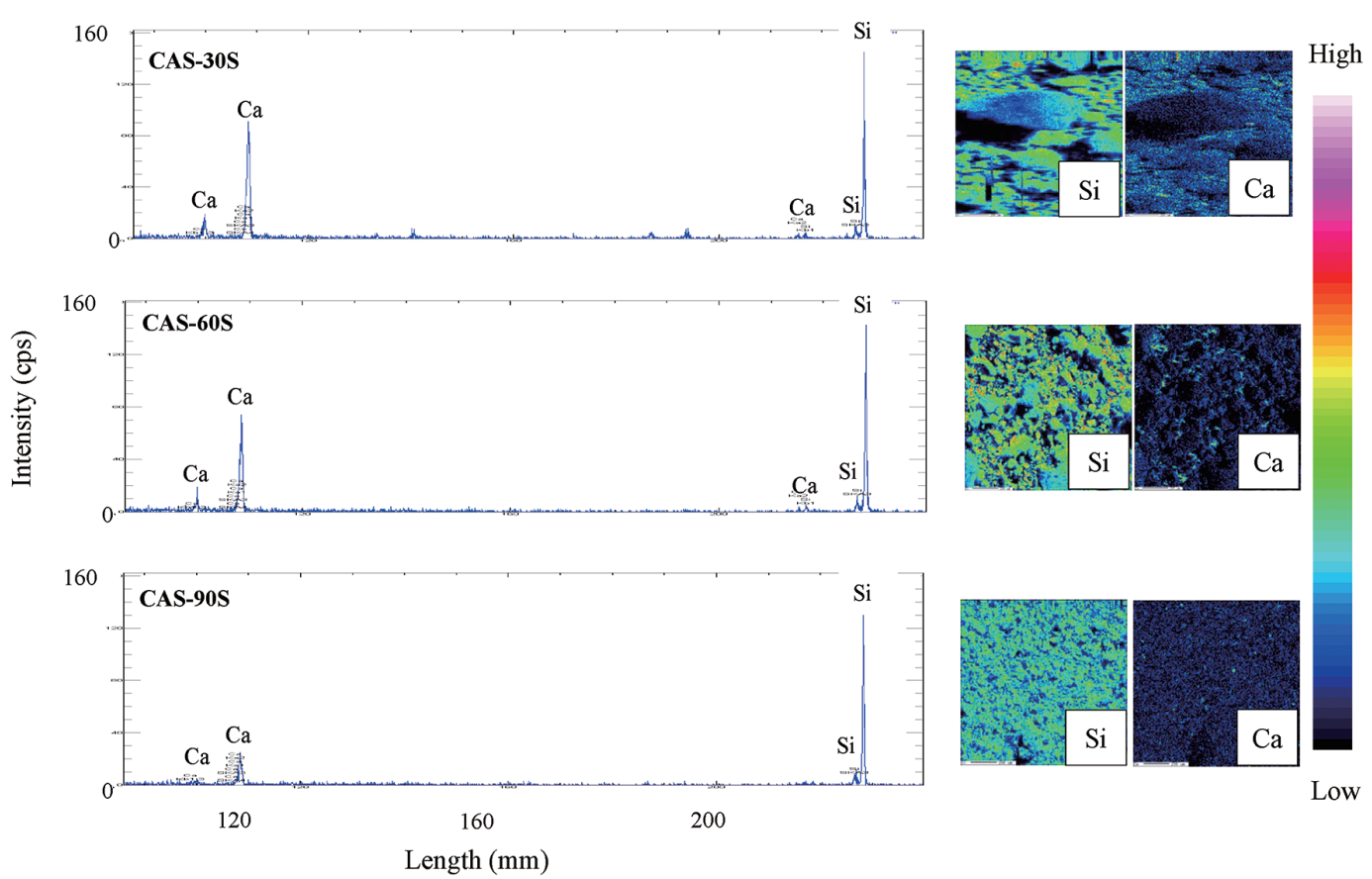

Fig. 2 Qualitative analysis of CASs surface.

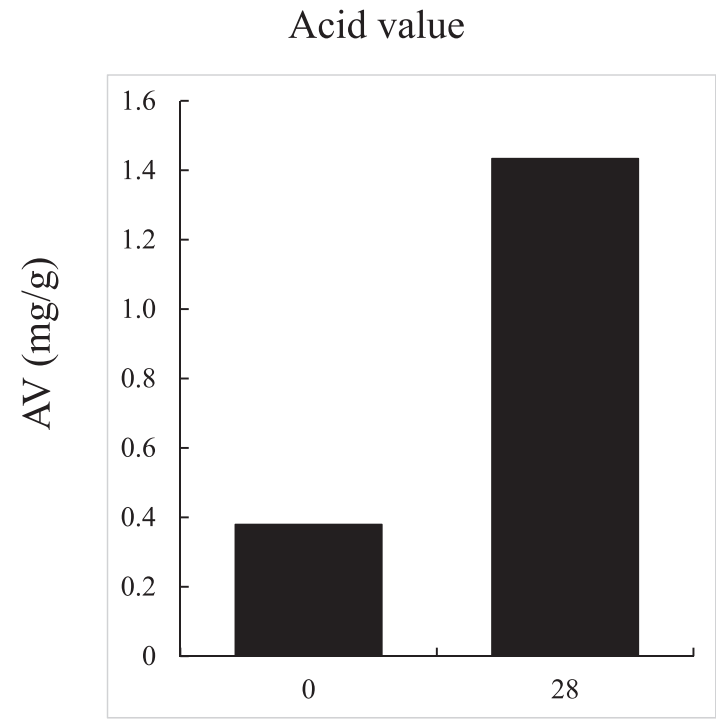

Time (hr)

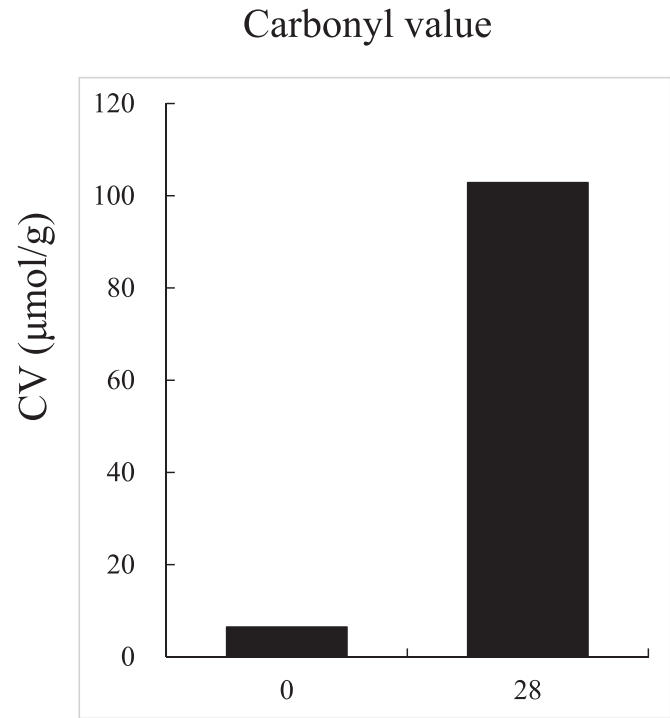

Time (hr)

Fig. 3 Changes in AV, and CV with deterioration treatment.

\subsection{Adsorbent treatment}

In previous work, FFAs from the nonaqueous phase were considered to be physisorbed on the adsorbent ${ }^{9}$. However, if a small amount of water was present on the adsorbent surface in the nonaqueous phase, the FFAs would be chemisorbed ${ }^{10)}$. These data indicate that the water in the nonaqueous oil phase is an important factor for the adsorption and removal of FFAs. In addition, some reports have stated that water was adhered onto a polar powder (adsorbent) in the nonaqueous phase ${ }^{11)}$, indicating that the adsorbent contains adhesive water and interacts with polar compounds in the nonaqueous phase.

In this study, we prepared an adsorbent treated with water. The decrease in AV and CV upon adsorbent treatment with water addition is shown in Fig. 4 . When the addition ratio of water in the adsorbent was $25 \%$ to $75 \%$, the $\mathrm{AV}$ decreased more significantly. However, the decrease in AV did not change when the water content in the adsorbent was above $75 \%$ (a significant difference was confirmed between CAS-30S (25\%) and CAS-90S (25 and 

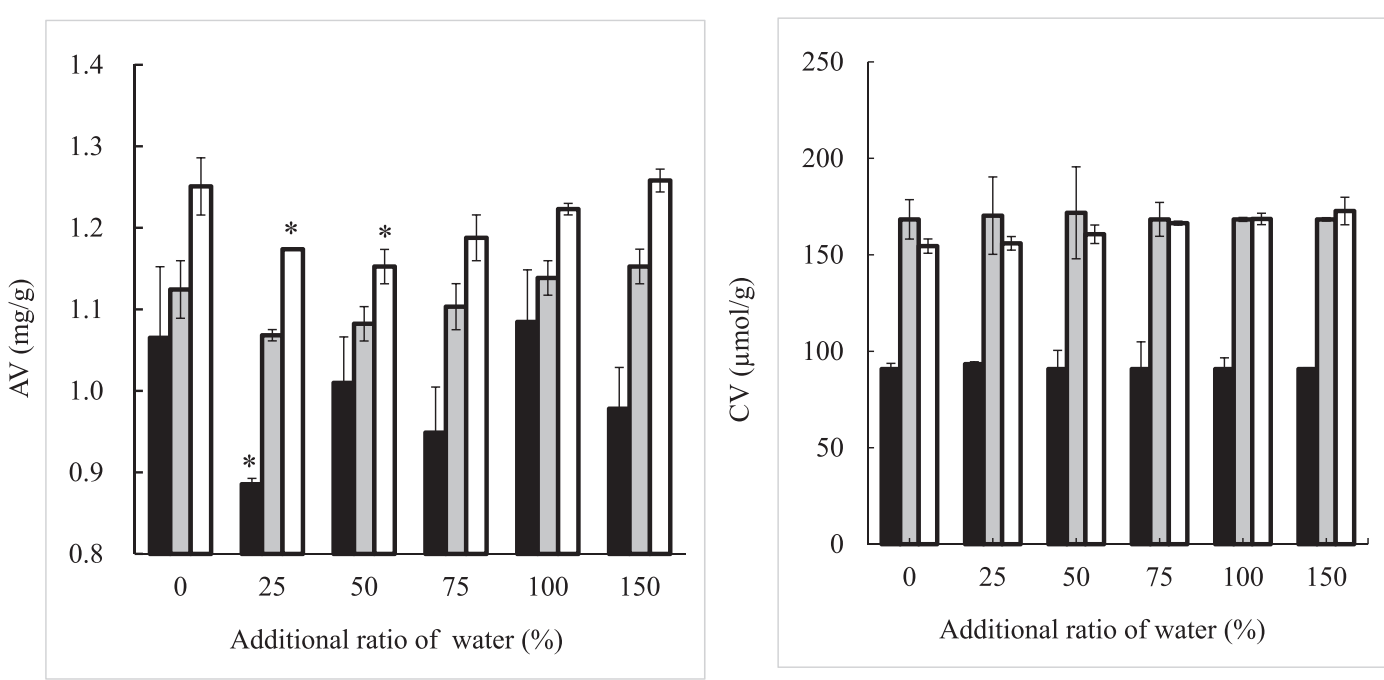

: CAS-30S, $\square:$ : CAS-60S, $\square$ : CAS-90S

( $p<0.05$ compared to $0 \%$ )

Fig. 4 Decreasing in AV and CV by CAS-30S, CAS-60S and CAS-90S treatment.

$50 \%)$ ) compared with the case when water was not added. On the other hand, the decrease in CV was not significant upon treatment with the adsorbent with water addition. The small amount of water on the adsorbent surface was attributed to the decrease of $\mathrm{AV}$ (not of CV), but excessive water addition on the adsorbent was attributed to increased deterioration of $\mathrm{SO}$, which indicated that the excessive water induces the degradation reaction (e.g. hydrolysis $)^{15)}$. These results indicate that adsorbent treatment with water addition is useful for the removal of $\mathrm{AV}$ from the nonaqueous phase. A previous study reported the effect of water addition on the adsorbent surface on the removal of chromaticity from the nonaqueous phase by activated clay $^{16)}$. The data obtained in this study show similar trends to those reported in the literature.

On the other hand, concentration of palmitic acid, stearic acid, oleic acid, linoleic acid, and linolenic acid before adsorbent treatment was $115.7 \pm 4.2 \mathrm{mg} / \mathrm{L}, 69.9 \pm$ $7.5 \mathrm{mg} / \mathrm{L}, 204.6 \pm 19.1 \mathrm{mg} / \mathrm{L}, 445.0 \pm 61.4 \mathrm{mg} / \mathrm{L}$, and $39.1 \pm$ $4.8 \mathrm{mg} / \mathrm{L}$, respectively. After adsorption treatment by CAS30S, CAS-60S, and CAS-90S (with an additional ratio of water of $0-150 \%)$, concentrations of the FFAs did not change drastically. Moreover, tocopherol $(\alpha-, \beta-, \gamma-$, and $\delta$ tocopherol) was not detected after deterioration under the experimental conditions used in this study.

As previously discussed, the adsorption of FFAs from the nonaqueous phase was attributed to physical adsorption". Therefore, the relationship between the decrease in AV and the specific surface area was investigated in this study (Fig. 5). The decrease in AV depended on the specific surface area, in agreement with previous studies ${ }^{9-11)}$. In addition, the adsorption mechanism of AV (polar compounds) onto water modified adsorbent in the nonaqueous

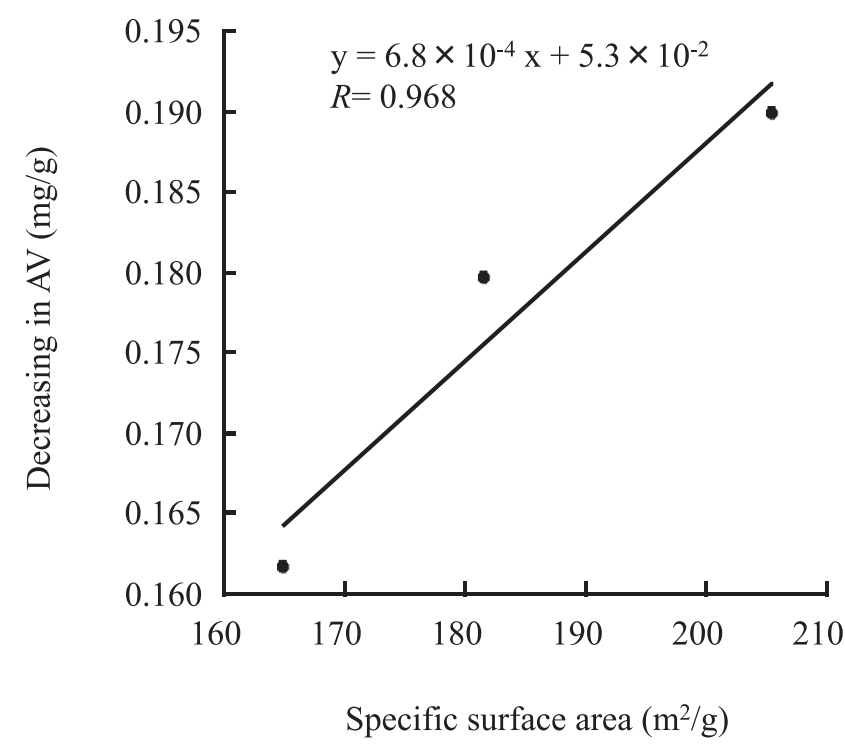

Fig. 5 Relationship between decreasing in AV and specific surface area.

phase was investigated(Fig. 6). A small amount of water addition on the adsorbent resulted in a water layer on adsorbent surface, which suggests that the adsorbent surface is polar. In addition, some calcium ions from the CAS moved to the polar layer in the adsorbent, and polar compounds subsequently adhered to the polar layer on the adsorbent surface from the nonaqueous phase. Finally, the polar compounds and calcium ions interacted with each other in the water layer via chemisorption. To confirm the mechanism described above, elemental composition of the adsorbent surface before and after adsorption treatment was evaluated. An increase in carbon content on the adsor- 


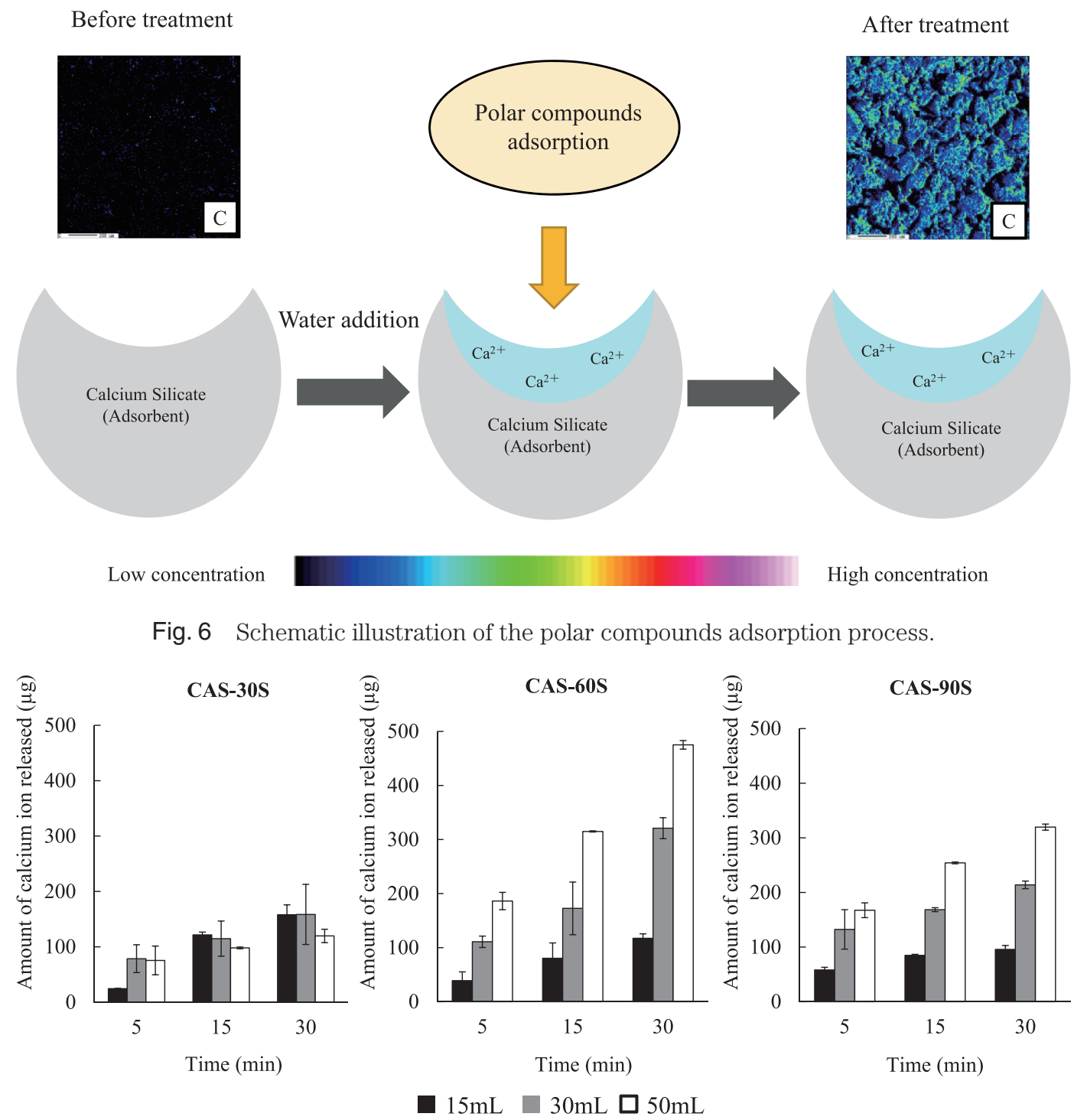

Fig. 7 Effect of amount water on releasing of calcium ion from CASs.

bent surface after adsorption treatment was confirmed (Fig. 6). These results indicate that polar organic compounds were adsorbed by the adsorbent surface treated with water addition.

Next, the effect of the amount water on the release of calcium ions from the adsorbent (CAS) was evaluated (Fig. 7). The adsorption mechanism of polar compounds on the adsorbent treated with water addition was related to calcium ion migration. Therefore, the concentration of calcium ions in the CAS was measured. The concentration of released calcium increased with increasing contact time and solvent volume. Figure 8 shows the relationship between the decrease in $\mathrm{AV}$ or $\mathrm{CV}$ and the amount of calcium ions released from the adsorbent. The correlation between decreasing $\mathrm{AV}$ and the amount of calcium released from the adsorbent was negative $(-0.975,-0.970$, and 0.927 for CAS30S, CAS-60S, and CAS-90S, respectively).
Moreover, the decrease in CV and the amount of calcium released from the adsorbent also showed negative relationship with the exception of CAS-30S $(-0.968$ and -0.969 for CAS-60S and CAS-90S). These results indicate that a small amount of water addition is suitable for preventing calcium ion loss from the adsorbent and for the removal of polar compounds in the nonaqueous phase.

\subsection{Application of adsorbent treatment with a small amount of water}

The data described above show that calcium can be released from the adsorbent. It is not clear whether the released calcium affects the deterioration of SO. Therefore, it is important to determine the effect of the calcium ions released from the adsorbent on the deterioration of SO. Figure 9 shows the changes in $\mathrm{AV}$ and $\mathrm{CV}$ with adsorbent treatment with water addition. After the $1^{\text {st }}$ and $2^{\text {nd }}$ adsorp- 

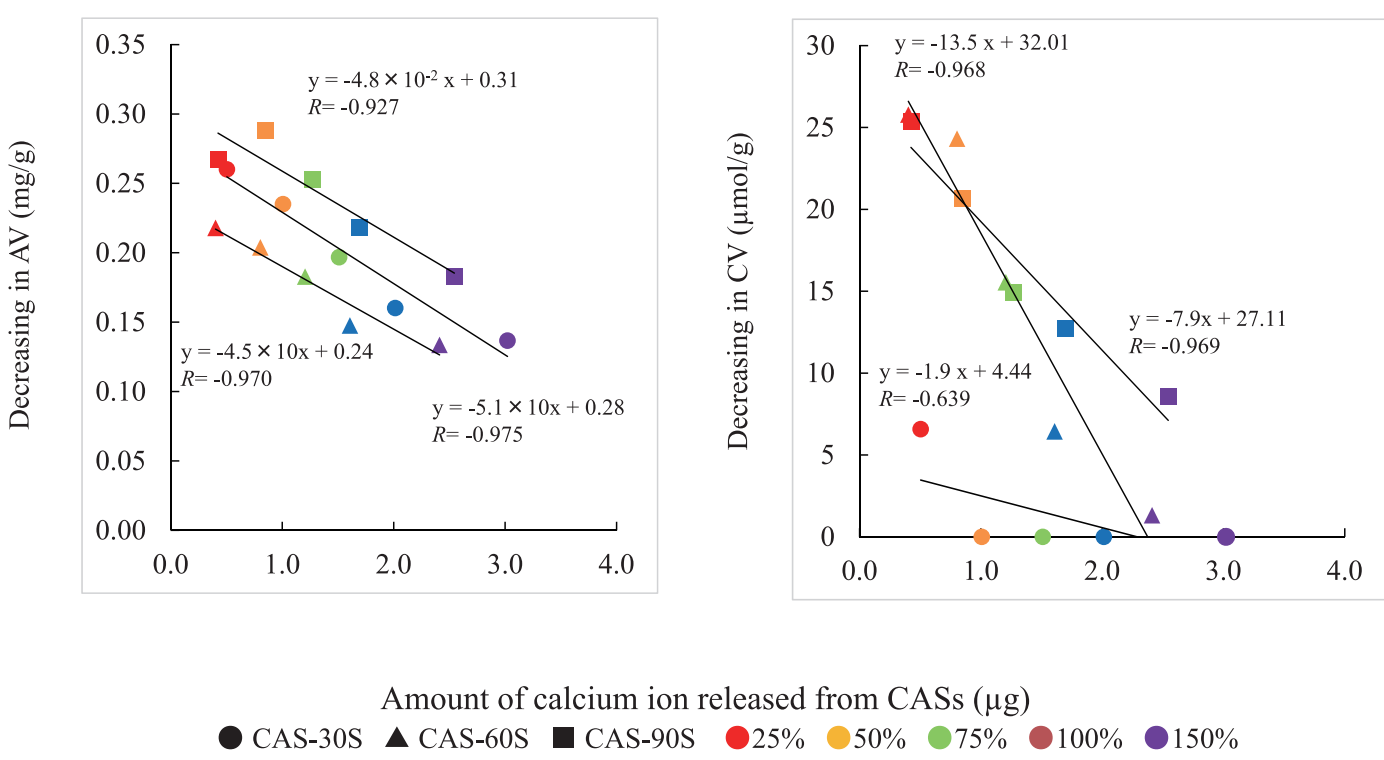

Fig. 8 Relationship between decreasing in AV or CV and the amount of calcium ion released from CASs.
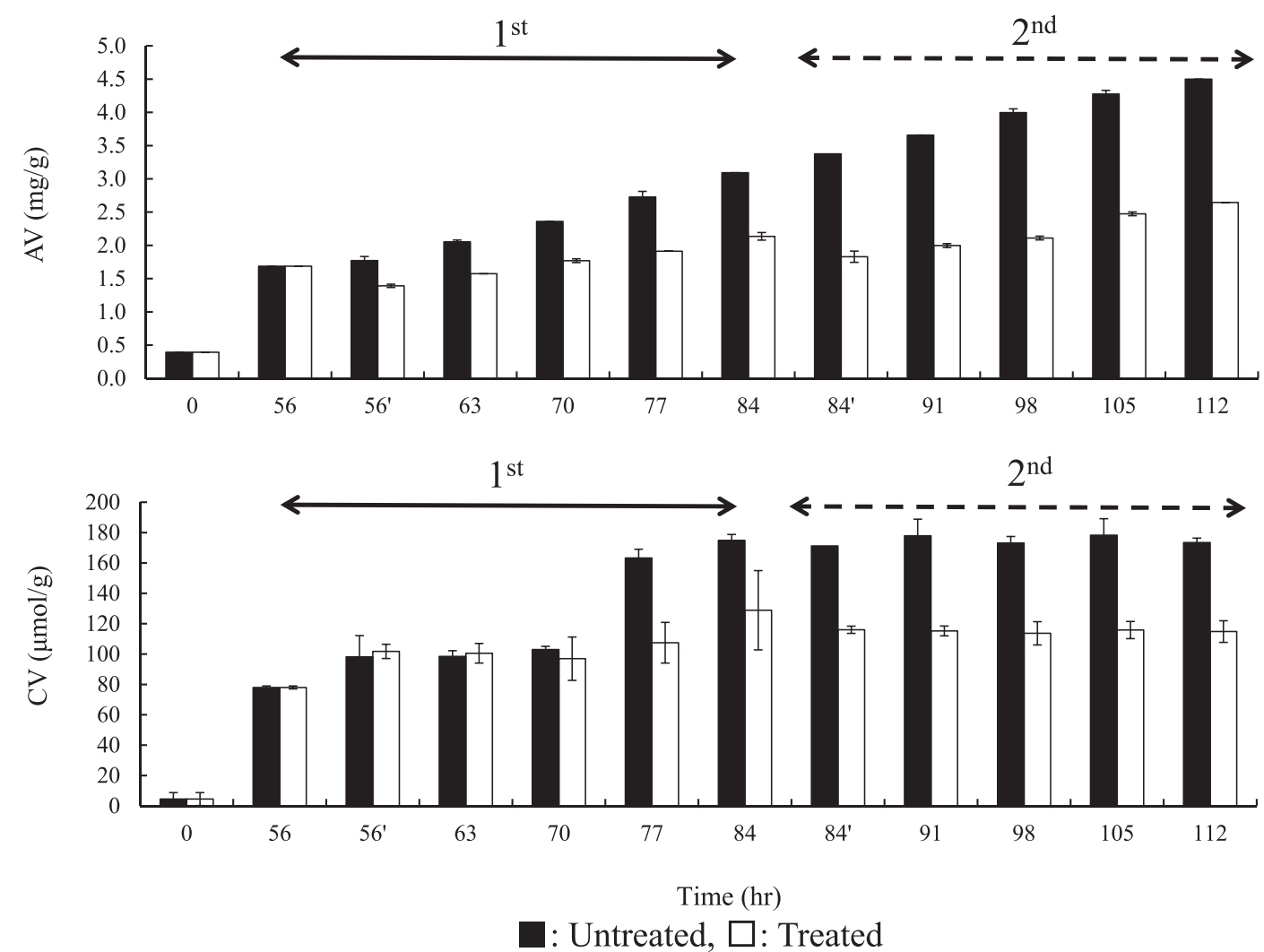

Fig. 9 Changes in AV and CV with adsorption treatment.

tion treatment, both $\mathrm{AV}$ and $\mathrm{CV}$ untreated with adsorbent were greater than those treated with adsorbent, which indicates that the calcium ion from the adsorbent did not affect the deterioration of SO under these experimental conditions. Figure 10 shows the AV of SO before and after adsorption treatment. The deterioration of untreated SO (slope is 0.05 and 0.04 ) is greater than that for SO after the $1^{\text {st }}$ and $2^{\text {nd }}$ adsorbent treatment (slope is 0.03 ). The CV in the $1^{\text {st }}$ and $2^{\text {nd }}$ adsorbent treatment did not change, which indicates that the calcium released from the adsorbent does not affect the CV. These data suggest that SO after adsorbent treatment is not easily deteriorated, and the adsorbent treatment is useful for preventing the deterioration of oil and could possibly contribute to the prolonging of the 

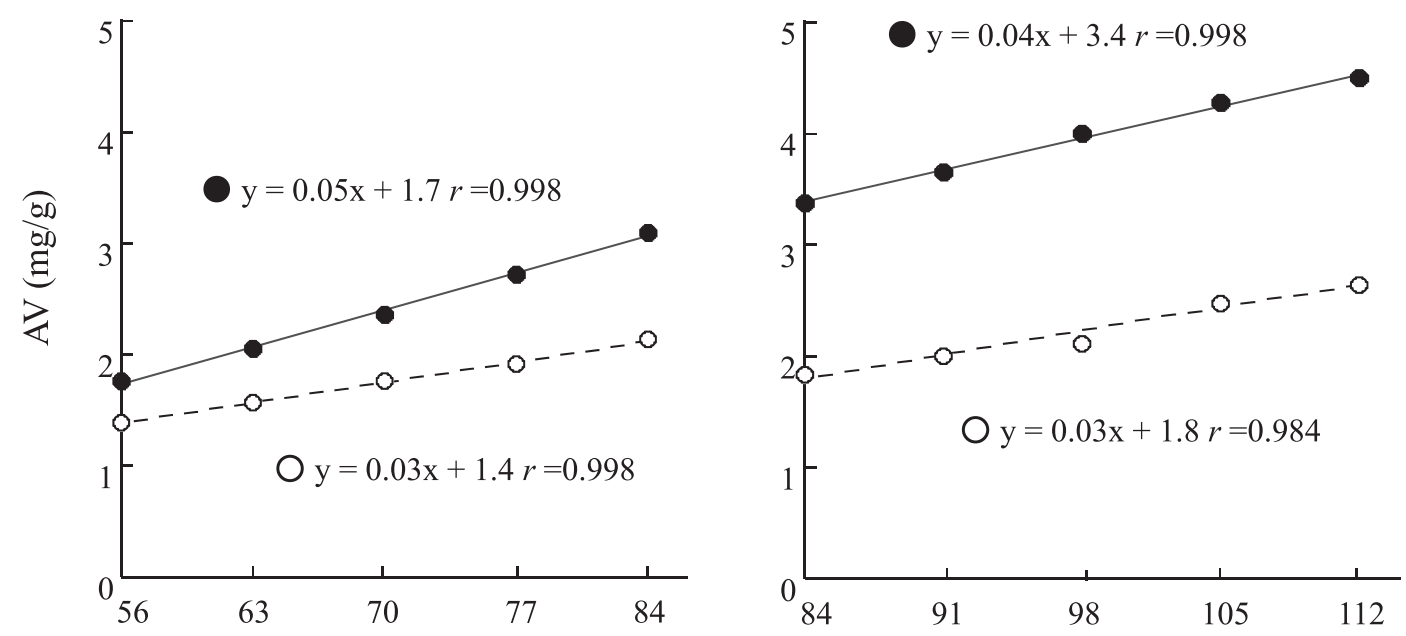

Time (hr)

: Untreated, O: Treated

Fig. $10 \mathrm{AV}$ in SO before and after adsorption treatment.

oil life cycle.

\section{Conclusion}

Calcium silicate adsorbents treated with different percentages of water were prepared in this study. The specific surface area and pore volume followed the order CAS-60S $<$ CAS-30S $<$ CAS-90S and CAS-90S $<$ CAS-60S $<$ CAS-30S, respectively. The heating and aeration combination treatment prepared deteriorated SO. The decrease in AV by treatment with CAS-30S (water addition $25 \%$ ) and CAS-90S (water addition 25 and $50 \%$ ) was amplified, however the rate of decrease of CV by adsorbent treatment was unchanged. The correlation between the decrease in $\mathrm{AV}$ and the specific surface area show a positive correlation (correlation coefficient; 0.968). A small amount of water addition on adsorbent surface forms a polar water layer, and subsequently polar compounds move to the water layer from the oil phase. Finally, the interaction between the water layer, containing released calcium ions from the adsorbent, and polar compounds in the oil occurred, suggesting that $\mathrm{AV}$ in the deteriorated SO decreased. The effect of water addition on preventing deterioration was also evaluated. The AV in deteriorated SO treated with adsorbent was smaller than in the untreated samples, and CV in deteriorated SO treated with adsorbent was unchanged. Collectively, the adsorbent treatment with water added to the adsorbent was useful for preventing deterioration of SO and could prolong the oil life cycle.

\section{Acknowledgment}

MEXT (Ministry of Education, Culture, Sports, Science and Technology)-supported Program for the Strategic Research Foundation at Private Universities, 2014-2018 (S1411037).

\section{References}

1) Sohaimi, K.S.A.; Ngadi, N.; Mat, H.; Inuwa, I.M.; Wong S. Synthesis, characterization and application of textile sludge biochars for oil removal. J. Environ. Chem. Eng. 5, 1415-1422 (2017).

2) Miyagi, A.; Nakajima, M. Regeneration of used frying oils using adsorption processing. J. Am. Oil Chem. Soc. 80, 91-96 (2003).

3) Gebhardt, B; Oils and fats in snack foods. in Bailey's Industrial Oil \& Fat Products. $5^{\text {th }}$ ed. (Hui, Y.H. ed.) Vol. 3, John Wiley \& Sons Press, New York, pp. 407427 (1996).

4) Turan, S.; Yalcuk, A. Regeneration of used frying oil. $J$. Am. Oil Chem. Soc. 90, 1761-1771(2013).

5) Lin, S.; Akoh, C.C.; Estes Reybolds, A. Determination of optimal conditions for selected adsorbent combinations to recover used frying oils. J. Am. Oil Chem. Soc. 76, 739-744 (1999).

6) Jacobson, G.A. Quality control of commercial deep-fat frying. Food Technol. 21, 43-48 (1967).

7) Sabah, E.; Celik, S.M. Sepiolite: An effective bleaching adsorbent for the physical refining of degummed rapeseed oil. J. Am. Oil Chem. Soc. 82, 911-916 (2005).

8) Ogata, F.; Kawasaki, N. Regeneration of waste edible oil by the use of virgin and calcined magnesium hy- 
droxide as adsorbents. J. Oleo Sci. 65, 941-948 (2016).

9) Yamada, T.; Komatsu, Y. Effect of adsorbed water onto activated clary and acid clay on decolorization in oil. YUSHIKAGAKU KYOUKAISHI 4, 191-193 (1995).

10) Nakagawa, M. Surface Chemistry (Fundamentals). II. YUKAGAKU 13, 148-155(1964).

11) Meguro, K.; Koishi, M. Water problems of powder surface. ZAIRYO 15, 133-137 (1966).

12) Pharmaceutical society of Japan, Hanyoueiseishikenhou to kaisetsu. Nanzando, Tokyo. p. 26 (1996).

13) Sato, Y.; Chiba, Y.; Kuzuoka, S.; Endo, M.; Kiyono, Y., Sasaki, T. Estimation of the deterioration of fried food by the carbonyl value using 1-butanol as a solvent. Annual report of Miyagi Prefectural Institute of Public Health and Environment 24, 136-138 (2006) .

14) Ogata, F.; Tanaka, Y.; Tominaga, H.; Kangawa, M.; Inoue, K.; Ueda, A.; Iwata, Y.; Kawasaki N. Study on analysis of waste edible oil with deterioration and removal of acid value, carbonyl value, and free fatty acid by a food additive (calcium silicate). J. Oleo Sci. 62, 109-114 (2013).

15) John, M.; Kakuda, Y.; Kamie, B. Improving the quality of used frying oils by treatment with activated carbon and silica. J. Am. Oil Chem. 63, 1564-1567 (1986).

16) Tachibana, T. Jpn. Pat. P5785179 (2015). 